\title{
AUTHOR INDEX \\ Volume 6 (2008)
}

Akiyama, Y., see Tsukamoto, K.

6 (2008) 1133

Aksianov, E., Zanegina, O., Grishin, A., Spirin, S., Karyagina, A. and Alexeevski, A., Conserved Water Molecules in X-ray Structures Highlight the Role of Water in Intramolecular and Intermolecular Interactions

Alexandrov, K., Sobolev, B., Filimonov, D. and Poroikov, V., Recognition of Protein Function Using the Local Similarity

Arnold, J., see Tewari, S.

Aung, Z., Tan, S.-H., Ng, S.-K. and Tan, K.-L., PPiClust:

Efficient Clustering of 3D Protein-Protein Interaction Interfaces

Bagos, P. G., see Litou, Z. I.

Baronova, T., see Zhu, Z.

4 (2008) 789

Baù, D., see Martin, A. J. M.

5 (2008) 1001

Bhandarkar, S. M., see Tewari, S.

1 (2008) 125

Bogatyreva, N. S., see Dovidchenko, N. V.

5 (2008) 1035

Bogatyreva, N. S., see Finkelstein, A. V.

4 (2008) 693

Bogatyreva, N. S., see Galzitskaya, O. V.

4 (2008) 667

Bouck, J., see Tatarinova, T.

Brodal, G. S., see Stissing, M.

1 (2008) 37

Bukhman, Y. V., Dharsee, M., Ewing, R., Chu, P., Topaloglou, T., Le Bihan, T., Goh, T., Duewel, H., Stewart, I. I.,

Wisniewski, J. R. and Ng, N. F., Design and Analysis of

Quantitative Differential Proteomics Investigations Using

LC-MS Technology

1 (2008) 107

Cary, S. C., see Kaplarevic, M.

6 (2008) 1193

Cedano, J., see Huerta, M.

2 (2008) 367

Choi, V., Huang, Y., Lam, V., Potter, D., Laubenbacher, R.

and Duca, K., Using Formal Concept Analysis for Microarray

Data Comparison

Chu, P., see Bukhman, Y. V. 
Chua, H. N., Ning, K., Sung, W.-K., Leong, H. W. and Wong, L., Using Indirect Protein-Protein Interactions for Protein Complex Prediction

3 (2008) 435

Cios, K. J., see Nguyen, C.

Close, T. J., see Wu, Y.

1 (2008) 203

3 (2008) 603

Comin, M., Guerra, C. and Zanotti, G., Mining Overrepresented 3D Patterns of Secondary Structures in Proteins

6 (2008) 1067

Coppée, J.-Y., see Osorio y Fortéa, J.

2 (2008) 317

Cui, F., Jernigan, R. and Wu, Z., Knowledge-based versus

Experimentally Acquired Distance and Angle Constraints for

NMR Structure Refinement

2 (2008) 283

2 (2008) 335

Danzer, J. F., see Poleksic, A.

2 (2008) 317

de Carvalho, Jr., S. A. and Rahmann, S., Better GeneChip

Microarray Layouts by Combining Probe Placement and

Embedding

3 (2008) 623

2 (2008) 335

4 (2008) 843

5 (2008) 933

Demin, O., see Peskov, K.

1 (2008) 107

1 (2008) 223

Dong, Y., see Fang, J.

4 (2008) 789

Dovidchenko, N. V., Bogatyreva, N. S. and Galzitskaya, O. V.,

5 (2008) 1035

Prediction of Loop Regions in Protein Sequence

4 (2008) 693

1 (2008) 65

1 (2008) 107

1 (2008) 1

Ellrott, K., Guo, J.-T., Olman, V. and Xu, Y., Improving the Performance of Protein Threading Using Insertion/Deletion Frequency Arrays

3 (2008) 585

3 (2008) 493

Engel, J. D., see Rao, A.

1 (2008) 107

1 (2008) 37

Fagerberg, R., see Stissing, M.

Fang, J., Dong, Y., Williams, T. D. and Lushington, G. H.,

Feature Selection in Validating Mass Spectrometry Database

Search Results

1 (2008) 223

5 (2008) 961

2 (2008) 335

Fienup, M., see Poleksic, A.

4 (2008) 709

Finkelstein, A. V., Lobanov, M. Y., Dovidchenko, N. V. and

Bogatyreva, N. S., Many-Atom van der Waals Interactions

Lead to Direction-Sensitive Interactions of Covalent Bonds 
Freier, S. M., see Sipes, T. B.

5 (2008) 919

$\mathrm{Fu}$, Z. and Jiang, T., Clustering of Main Orthologs for Multiple Genomes

3 (2008) 573

Fujita, A., Sato, J. R., Garay-Malpartida, H. M., Sogayar, M.

C., Ferreira, C. E. and Miyano, S., Modeling Nonlinear Gene

Regulatory Networks from Time Series Gene Expression Data

Fukui, K., see Tsukamoto, K.

5 (2008) 961

6 (2008) 1133

Galzitskaya, O. V., Bogatyreva, N. S. and Ivankov, D. N.,

Compactness Determines Protein Folding Type

4 (2008) 667

5 (2008) 1035

Galzitskaya, O. V., Search for Folding Initiation Sites from

Amino Acid Sequence

4 (2008) 681

Gamalielsson, J. and Olsson, B., Gene Ontology-based Semantic

Alignment of Biological Pathways by Evolutionary Search

Gao, G. R., see Kaplarevic, M.

Gao, Y., see Zhu, Z.

Garay-Malpartida, H. M., see Fujita, A.

Gardiner, K., see Nguyen, C.

Gaur, D. R., see Maňuch, J.

Goh, T., see Bukhman, Y. V.

4 (2008) 825

6 (2008) 1193

4 (2008) 789

5 (2008) 961

1 (2008) 203

1 (2008) 93

Gondo, Y., see Kaminuma, E.

1 (2008) 107

5 (2008) 905

Goryanin, I., see Peskov, K.

4 (2008) 843

5 (2008) 933

Goryanin, I., see Peskov, K.

5 (2008) 885

Greenberg, J., see Tsigelny, I. F.

4 (2008) 775

Grishin, A., see Aksianov, E.

6 (2008) 1067

Guerra, C., see Comin, M.

3 (2008) 585

Guo, J.-T., see Ellrott, K.

6 (2008) 1157

Hackermüller, J., see Rose, D.

2 (2008) 387

Hamodrakas, S. J., see Litou, Z. I.

6 (2008) 1177

Harner, E. J., see Sazonova, N.

1 (2008) 183

2 (2008) 261

He, W., see Yuan, A.

3 (2008) 493

6 (2008) 1115

1 (2008) 1

4 (2008) 747

6 (2008) 1133

1 (2008) 65

Huang, Y., see Choi, V.

Huerta, M., Cedano, J. and Querol, E., Analysis of Nonlinear

Relations Between Expression Profiles by the Principal

Curves of Oriented-Points Approach

2 (2008) 367

3 (2008) 543

Husmeier, D., see Werhli, A. V.

4 (2008) 667

Ivankov, D. N., see Galzitskaya, O. V. 
Ivliev, A. E. and Sergeeva, M. G., OrthoFocus: Program for Identification of Orthologs in Multiple Genomes in

Family-Focused Studies

4 (2008) 811

Jernigan, R., see Cui, F.

2 (2008) 283

Jiang, M., Xu, Y. and Zhu, B., Protein Structure-Structure

Alignment with Discrete Fréchet Distance

$1(2008) 51$

Jiang, T., see Fu, Z.

3 (2008) 573

Jiang, T., see Li, J.

1 (2008) 241

Jöris, J., see Rose, D.

6 (2008) 1157

Kaminuma, E., Masuya, H., Miura, I., Motegi, H., Takahasi, K.

R., Nakazawa, M., Matsui, M., Gondo, Y., Noda, T.,

Shiroishi, T., Wakana, S. and Toyoda, T., Objective

Evaluation Measures of Genetic Marker Selection in

Large-Scale SNP Genotyping

Kaplarevic, M., Murray, A. E., Cary, S. C. and Gao, G. R.,

EnGenIUS - Environmental Genome Informational Utility

System

6 (2008) 1193

Karyagina, A., see Aksianov, E.

4 (2008) 775

Klebanov, L., Qiu, X. and Yakovlev, A., Testing Differential

Expression in Nonoverlapping Gene Pairs: A New Perspective for the Empirical Bayes Method

Kouznetsova, V., see Tsigelny, I. F.

2 (2008) 301

5 (2008) 885

1 (2008) 77

Lajoie, G., see Shan, B.

5 (2008) 1021

Lam, T.-W., see Wong, T. K. F.

1 (2008) 65

Lang, T., see Osorio y Fortéa, J.

2 (2008) 317

Laubenbacher, R., see Choi, V.

1 (2008) 65

Le Bihan, T., see Bukhman, Y. V.

1 (2008) 107

Lebedev, Y. B., see Panchin, A. Y.

4 (2008) 759

3 (2008) 435

Leong, H. W., see Chua, H. N.

3 (2008) 467

Li, J. and Jiang, T., A Survey on Haplotyping Algorithms for

Tightly Linked Markers

1 (2008) 241

3 (2008) 521

Li, M., see Zhang, Z.

6 (2008) 1157

Li, Q., see Rose, D.

6 (2008) 1089

Li, W., see Liu, Y.

2 (2008) 387

5 (2008) 981

Lin, G., see Wan, X.-F.

3 (2008) 521

Litou, Z. I., Bagos, P. G., Tsirigos, K. D., Liakopoulos, T. D. and Hamodrakas, S. J., Prediction of Cell Wall Sorting Signals in Gram-Positive Bacteria with a Hidden Markov Model: Application to Complete Genomes 
Liu, L., see Wu, Y.

3 (2008) 603

Liu, Y. and Li, W., Visualizing Microarray Data for Biomarker Discovery by Matrix Reordering and Replicator Dynamics

Lobanov, M. Y., see Finkelstein, A. V.

Lonardi, S., see $\mathrm{Wu}, \mathrm{Y}$.

6 (2008) 1089

4 (2008) 693

3 (2008) 603

Lonardi, S., see Wu, Y.

6 (2008) 1049

Lu, Y., He, J. and Strauss, C. E. M., Deriving Topology and Sequence Alignment for the Helix Skeleton in Low-Resolution Protein Density Maps

1 (2008) 183

4 (2008) 759

Lukyanov, S. A., see Panchin, A. Y.

1 (2008) 223

1 (2008) 77

Ma, B., see Shan, B.

1 (2008) 37

1 (2008) 203

Mannino, M., see Nguyen, C.

Maňuch, J. and Gaur, D. R., Fitting Protein Chains to Cubic Lattice Is NP-Complete

1 (2008) 93

Martin, A. J. M., Baù, D., Vullo, A., Walsh, I. and Pollastri, G., Long-Range Information and Physicality Constraints Improve Predicted Protein Contact Maps

5 (2008) 1001

Masuya, H., see Kaminuma, E.

5 (2008) 905

5 (2008) 905

Miao, X., Waddell, P. J. and Valafar, H., TALI: Local

Alignment of Protein Structures Using Backbone Torsion Angles

1 (2008) 163

2 (2008) 317

Milon, G., see Osorio y Fortéa, J.

5 (2008) 905

Miura, I., see Kaminuma, E.

5 (2008) 961

Miyano, S., see Fujita, A.

5 (2008) 905

Motegi, H., see Kaminuma, E.

Murray, A. E., see Kaplarevic, M.

6 (2008) 1193

Nakazawa, M., see Kaminuma, E.

5 (2008) 905

Ng, N. F., see Bukhman, Y. V.

1 (2008) 107

Ng, S.-K., see Aung, Z.

3 (2008) 415

Nguyen, C., Mannino, M., Gardiner, K. and Cios, K. J., ClusFCM: An Algorithm for Predicting Protein Functions Using Homologies and Protein Interactions

1 (2008) 203

Nguyen, T.-P. and Ho, T.-B., An Integrative Domain-based Approach to Predicting Protein-Protein Interactions

6 (2008) 1115

5 (2008) 885

3 (2008) 435

Ning, K., see Chua, H. N.

Ning, K., Ye, N. and Leong, H. W., On Preprocessing and Antisymmetry in De Novo Peptide Sequencing: Improving Efficiency and Accuracy

Noda, T., see Kaminuma, E.

5 (2008) 905 
Olman, V., see Ellrott, K.

3 (2008) 585

Olsson, B., see Gamalielsson, J.

4 (2008) 825

Orshansky, I., see Sinitsina, N.

4 (2008) 869

Osorio y Fortéa, J., Prina, É., Lang, T., Milon, G., Davory, C.,

Coppée, J.-Y. and Regnault, B., AffyGCQC: A Web-based

Interface to Detect Outlying GeneChips with Extreme

Studentized Deviate Tests

2 (2008) 317

O'Toole, N., see Zhu, Z.

4 (2008) 789

Ozden, M., see Wan, X.-F.

5 (2008) 981

Panchin, A. Y., Spirin, S. A., Lukyanov, S. A., Lebedev, Y. B. and Panchin, Y. V., Human Trash ESTs - Sequences from cDNA Collection That Are Not Aligned to Genome Assembly 4 (2008) 759

Panchin, Y. V., see Panchin, A. Y.

4 (2008) 759

Pedersen, C. N. S., see Stissing, M.

Peskov, K., Goryanin, I. and Demin, O., Kinetic Model of Phosphofructokinase-1 from Escherichia coli

$1(2008) 37$

$4(2008) 843$

Peskov, K., Goryanin, I., Prank, K., Tobin, F. and Demin, O., Kinetic Modeling of ace Operon Genetic Regulation in Escherichia coli

5 (2008) 933

Pokrzywa, R., New Method for Yeast Identification Using Burrows-Wheeler Transform

Poleksic, A., Fienup, M., Danzer, J. F. and Debe, D. A., A Different Look at the Quality of Modeled Three-Dimensional Protein Structures

2 (2008) 335

5 (2008) 1001

Pollastri, G., see Martin, A. J. M.

4 (2008) 709

1 (2008) 65

5 (2008) 933

Prank, K., see Peskov, K.

2 (2008) 317

2 (2008) 301

Qiu, X., see Klebanov, L.

2 (2008) 367

Raghupathy, N., Hoberman, R. and Durand, D., Two Plus Two Does Not Equal Three: Statistical Tests for Multiple Genome Comparison

$1(2008) 1$

Rahmann, S., see de Carvalho, Jr., S. A.

3 (2008) 623

Rao, A., Hero, A. O. III, States, D. J. and Engel, J. D., Using

Directed Information to Build Biologically Relevant Influence

Networks

Regnault, B., see Osorio y Fortéa, J.

6 (2008) 1157

Rose, D., Jöris, J., Hackermüller, J., Reiche, K., Li, Q. and

Stadler, P. F., Duplicated RNA Genes in Teleost Fish

Genomes

6 (2008) 1157 
Sato, J. R., see Fujita, A.

5 (2008) 961

Sazonova, N. and Harner, E. J., Haplotype Inference and Block

Partitioning in Mixed Population Samples

6 (2008) 1177

Schumitzky, A., see Tatarinova, T.

Sergeeva, M. G., see Ivliev, A. E.

4 (2008) 727

4 (2008) 811

Shan, B., Ma, B., Zhang, K. and Lajoie, G., Complexities and Algorithms for Glycan Sequencing Using Tandem

Mass Spectrometry

1 (2008) 77

5 (2008) 905

4 (2008) 643

Shkuropatov, A. Ya., see Yakovlev, A. G.

4 (2008) 643

4 (2008) 643

Shuvalov, V. A., see Yakovlev, A. G.

Sinitsina, N., Orshansky, I. and Sokolova, O., Actin-Binding

Proteins: How to Reveal the Conformational Changes

$4(2008) 869$

Sipes, T. B. and Freier, S. M., Prediction of Antisense

Oligonucleotide Efficacy Using Aggregate Motifs

5 (2008) 919

Sobolev, B., see Alexandrov, K.

Sogayar, M. C., see Fujita, A.

4 (2008) 709

5 (2008) 961

Sokolova, O., see Sinitsina, N.

4 (2008) 869

Spirin, S., see Aksianov, E.

4 (2008) 775

Spirin, S. A., see Panchin, A. Y.

4 (2008) 759

Stadler, P. F., see Rose, D.

6 (2008) 1157

3 (2008) 493

States, D. J., see Rao, A.

1 (2008) 107

Stissing, M., Mailund, T., Pedersen, C. N. S., Brodal, G. S. and Fagerberg, R., Computing the All-Pairs Quartet Distance on a Set of Evolutionary Trees

Strauss, C. E. M., see Lu, Y.

Sung, W.-K., see Chua, H. N.

Takahasi, K. R., see Kaminuma, E.

Tan, K.-L., see Aung, Z.

Tan, S.-H., see Aung, Z.

Tatarinova, T., Bouck, J. and Schumitzky, A., Kullback-Leibler Markov Chain Monte Carlo - A New Algorithm for Finite

Mixture Analysis and Its Application to Gene Expression Data

Tewari, S., Arnold, J. and Bhandarkar, S. M., Likelihood of a

Particular Order of Genetic Markers and the Construction of

Genetic Maps

1 (2008) 37

1 (2008) 183

3 (2008) 435

5 (2008) 905

3 (2008) 415

3 (2008) 415

Tobin, F., see Peskov, K.

1 (2008) 125

5 (2008) 933

Tominaga, D. and Horimoto, K., Judgment Algorithm for

Periodicity of Time Series Data Based on Bayesian

Information Criterion

4 (2008) 747 
Topaloglou, T., see Bukhman, Y. V.

1 (2008) 107

Tovchigrechko, A., see Zhu, Z.

4 (2008) 789

Toyoda, T., see Kaminuma, E.

5 (2008) 905

Tsigelny, I. F., Greenberg, J., Kouznetsova, V. and Nigam, S.

K., Modeling of Glycerol-3-Phosphate Transporter Suggests a

Potential 'Tilt' Mechanism Involved in Its Function

5 (2008) 885

Tsirigos, K. D., see Litou, Z. I.

2 (2008) 387

Tsukamoto, K., Yoshikawa, T., Hourai, Y., Fukui, K. and

Akiyama, Y., Development of an Affinity Evaluation and

Prediction System by Using the Shape Complementarity

Characteristic Between Proteins

6 (2008) 1133

Vakser, I. A., see Zhu, Z.

4 (2008) 789

Valafar, H., see Miao, X.

1 (2008) 163

Vasilieva, L. G., see Yakovlev, A. G.

4 (2008) 643

Vullo, A., see Martin, A. J. M.

5 (2008) 1001

Waddell, P. J., see Miao, X.

1 (2008) 163

5 (2008) 905

Wakana, S., see Kaminuma, E.

5 (2008) 1001

Wan, X.-F., Ozden, M. and Lin, G., Ubiquitous Reassortments in Influenza A Viruses

5 (2008) 981

Wang, S. and Zheng, W.-M., CLePAPS: Fast Pair Alignment of Protein Structures Based on Conformational Letters

$2(2008) 347$

Werhli, A. V. and Husmeier, D., Gene Regulatory Network

Reconstruction by Bayesian Integration of Prior Knowledge and/or Different Experimental Conditions

3 (2008) 543

Williams, T. D., see Fang, J.

1 (2008) 223

1 (2008) 107

3 (2008) 435

Wong, L., see Chua, H. N.

Wong, T. K. F., Lam, T.-W., Yang, W. and Yiu, S. M., Finding Alternative Splicing Patterns with Strong Support from Expressed Sequences on Individual Exons/Introns

5 (2008) 1021

Wu, Y., Liu, L., Close, T. J. and Lonardi, S., Deconvoluting BAC-Gene Relationships Using a Physical Map

3 (2008) 603

Wu, Y. and Lonardi, S., A Linear-Time Algorithm for

Predicting Functional Annotations from PPI Networks

6 (2008) 1049

$\mathrm{Wu}, \mathrm{Z}$., see Cui, F.

2 (2008) 283

$\mathrm{Xu}, \mathrm{W}$. , The Distribution of Distances Between Randomly

Constructed Genomes: Generating Function, Expectation,

Variance and Limits

$1(2008) 23$

$\mathrm{Xu}, \mathrm{Y}$. , see Ellrott, K.

3 (2008) 585

$\mathrm{Xu}, \mathrm{Y}$., see Jiang, M.

1 (2008) 51

Yakovlev, A., see Klebanov, L.

2 (2008) 301 
Yakovlev, A. G., Shkuropatova, T. A., Vasilieva, L. G., Shkuropatov, A. Ya. and Shuvalov, V. A., Wave Packet Motions Coupled to Electron Transfer in Reaction Centers of Chloroflexus Aurantiacus

4 (2008) 643

Yang, W., see Wong, T. K. F.

5 (2008) 1021

Ye, N., see Ning, K.

3 (2008) 467

Yiu, S. M., see Wong, T. K. F.

5 (2008) 1021

Yoshikawa, T., see Tsukamoto, K.

6 (2008) 1133

Yuan, A. and He, W., Semiparametric Clustering Method for Microarray Data Analysis

Zanegina, O., see Aksianov, E.

2 (2008) 261

4 (2008) 775

6 (2008) 1067

Zanotti, G., see Comin, M.

1 (2008) 77

Zhang, Z., Lin, H. and Li, M., MANGO: Multiple Alignment with N Gapped Oligos

3 (2008) 521

Zheng, W.-M., see Wang, S.

2 (2008) 347

Zhu, B., see Jiang, M.

1 (2008) 51

Zhu, Z., Tovchigrechko, A., Baronova, T., Gao, Y., Douguet, D., O'Toole, N. and Vakser, I. A., Large-Scale Structural Modeling of Protein Complexes at Low Resolution

4 (2008) 789 